\title{
Applied Metrology In The Production Of Supercon- ducting Model Magnets For Particle Accelerators
}

\author{
J. Ferradas Troitino, P. Bestmann, N. Bourcey, A. Carlon Zurita, E. Cavanna, P. Ferracin, S. Ferradas Troitino, E. F. \\ Holik, S. Izquierdo Bermudez, F. Lackner, C. Löffler, G. Maury, J. C. Perez, F. Savary, M. Semeraro and G. Vallone
}

\begin{abstract}
The production of superconducting magnets for particle accelerators involves high precision assemblies and tight tolerances, in order to achieve the requirements for their appropriate performance. It is therefore essential to have a strict control and traceability over the geometry of each component of the system, and also to be able to compensate possible inherent deviations coming from the production process.

The objective of this paper is to present the experience from systematic geometrical measurements performed during the on-going production of model magnets for the High Luminosity - LHC upgrade. First, the methodology for the data acquisition and its ulterior analysis is described. Then, the results obtained in terms of coil geometry are explained with the goal of identifying the principal factors causing systematic and unexpected dimensional deviations. Finally, the integrated effect of assembly operations, cool down and powering of the magnet is investigated looking at measurements before and after cold tests.
\end{abstract}

Index Terms - Geometrical measurements, metrology, superconducting model magnets for particle accelerators, MQXFS, 11 $\mathrm{T}$ dipole, HL-LHC models.

\section{INTRODUCTION}

$\mathbf{S}$ UPERCONDUCTING magnets for particle accelerators are designed to provide, on each of their applications, the desired magnetic field needed for the interaction with the particle beam. For correct operation, the relative field imperfections in the main magnets should not exceed, in general, a few parts in $10^{4}$ compared to a pure ideal field [1].

This high field quality must be maintained during magnet powering, when electromagnetic forces act on the current carrying elements and tend to deform the system.

Both requirements are achieved, firstly by high positional accuracies for the current conductors and secondly, with sound designed magnet structures that deal with the electromagnetic forces.

Manuscript receipt and acceptance dates will be inserted here. Acknowledgment of support is placed in this paragraph as well. Consult the IEEE Editorial Style Manual for examples. This work was supported by the IEEE Council on Superconductivity under contract No. ABCD-123456789.

This work was supported by the High Luminosity LHC Project at CERN and by the DOE through the U.S. LHC Accelerator Research Program.

J. Ferradas Troitino, P. Bestmann, N. Bourcey, A. Carlon Zurita, E. Cavanna, P. Ferracin, S. Ferradas Troitino, S. Izquierdo Bermudez, F. Lackner, C. Löffler, G. Maury, J. C. Perez, F. Savary, M. Semeraro and G. Vallone are with CERN, 1211 Geneva, Switzerland (e-mail: jose.ferradas.troitino@cern.ch).

E.F. Holik is with Fermi National Accelerator Laboratory, Batavia, IL 80510 USA.

Color versions of one or more of the figures in this paper are available online at http://ieeexplore.ieee.org.

Digital Object Identifier will be inserted here upon acceptance.

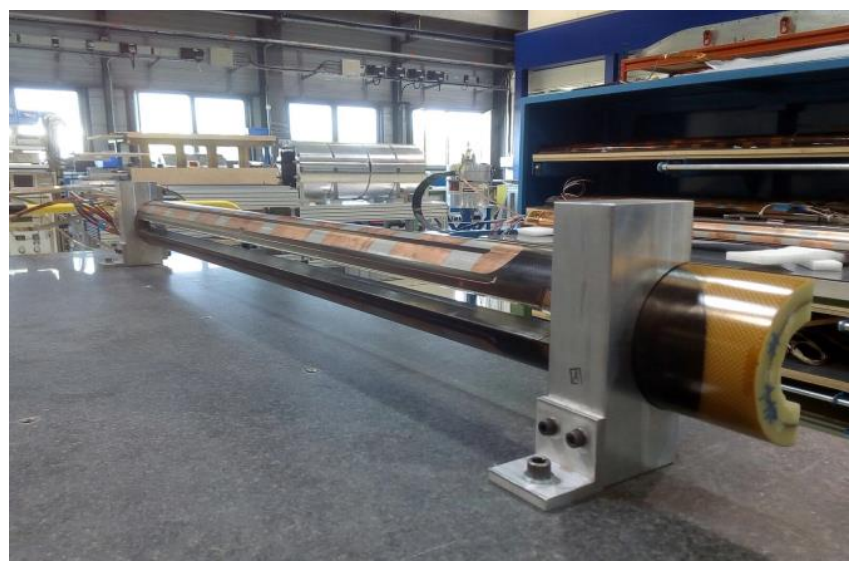

Fig. 1. HL-LHC 11 T Dipole coil fixed on the marble reference surface, ready for geometrical measurements.

On the first case, the required positional accuracy for conductor placement is usually in the order of few tens of micrometres. To reach this design target, structural components and superconducting coils must be manufactured ensuring their precise dimensioning. Systematic geometrical checks must be performed during the production phase in order to guarantee the requirements.

At CERN, dimensional measurements were performed for the dipole and quadrupole coils installed in the Large Hadron Collider [2]. Nowadays, the new LHC luminosity upgrade [3], [4] has required the development of two new superconducting magnets wound with $\mathrm{Nb}_{3} \mathrm{Sn}$ superconductor: the $11 \mathrm{~T}$ dipole for the arcs [5] and the MQXF quadrupole for the insertion regions [6]. These two projects are already well advanced in the prototyping phase, after having gone through a short model development program [7], [8]. At the moment of writing this paper, a total of 17 model coils have been produced for each of the projects. Valuable data about coil geometry and its variation during the development phase can be extracted from the analysis of this coil population. In this purpose, the model coils from both projects have been systematically measured using a commercial portable Coordinate Measurement Machine (CMM) equipped with a tactile probe. From the three dimensional point cloud generated, the coil geometry has been analysed and finally discussed along production.

An introduction to the measurements campaign and the results obtained is presented here. 


\section{Methodology}

\section{A. Geometrical Measurements Device}

As previously mentioned, the geometrical measurements presented on this paper have been carried out using a commercial portable Coordinate Measurement Machine (CMM). The technical specification for the machine used is listed below [9]:

- Volumetric Accuracy: $\pm 41 \mu \mathrm{m}$.

- Single Point Repeatability: $29 \mu \mathrm{m}$.

Technical specification values are obtained using a subset of test methods given in the ASME B89.4.22 standard [10].

\section{B. Geometrical Measurements Setup and Procedure}

All superconducting coils are fixed on a marble reference surface using dedicated fixtures. The supporting system is designed to facilitate the data acquisition (See Fig. 1).

The same measurements procedure is used for both HLLHC magnets in order to have a common frame for the analysis of the results.

The geometry of the coils is recreated using a dense point cloud divided in two categories: global data and cross sectional data. Fig. 2 shows the CAD model and data point cloud for a HL-LHC 11 T Dipole model coil.

- The global data is defined as the group of points that delimits the whole external envelope of the coil. It is used for the analysis of the overall geometry.

- The cross sectional data is defined by the acquisition of data points mapping transverse coil outlines in multiple locations along the longitudinal length of the coil. This data leads to the analysis of the coil geometry as function of the longitudinal length.

The actual coil geometry is always compared to the nominal design one, and results are usually given in terms of deviations rather than absolute values. To do so, nominal CAD models are employed as reference and the real geometry is compared to them. This comparison is based on best-fit algorithms that are used for the alignment between the acquired data points and the nominal CAD geometry.

The alignment is set to reproduce the functional magnet configuration. Each cross section is handled independently and aligned using the relevant features that define the position of the coil inside the structure. The used best-fit algorithm applies the same unitary weighting function to all the points considered for the alignment, around 600 per cross section in both coils, of which the $5 \%$ of outliers are excluded.

For the $11 \mathrm{~T}$ dipole, a total of 18 cross sections divided in left and right are measured, each one containing approximately 1000 data points. The alignment is performed using the outer diameter and loading plate points (See Fig. 3).

For the MQXFS quadrupole, 11 cross sections are measured. The alignment is done using the outer diameter and keyway points. The number of points per cross section is close to 1500 points in this case.

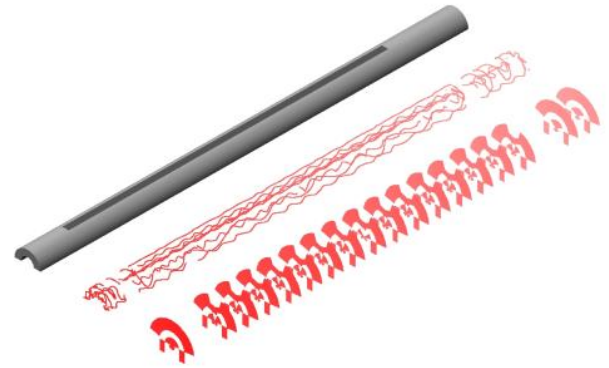

Fig. 2. HL-LHC 11 T Dipole coil CAD model and data point cloud.

\section{Analysis Convention}

Due to the alignment selected, deviations are grouped at the coil mid-planes and inner radius. For the complete description of coil geometry and the fair comparison between coils, the following parameters have been defined:

- Coil azimuthal size: Defined as the overall deviation in azimuthal direction, it is obtained as the sum of both mid-planes deviation values.

$$
\text { Size }=\mathrm{L}+\mathrm{R}
$$

- Coil asymmetry: Describing the asymmetry of the coil mid-planes, it is defined as the difference between the left mid-plane deviation and the right mid-plane one.

$$
\text { Asymmetry = L-R }
$$

- Coil radial size or coil width: It is expressed as the deviation from the nominal geometry in radial direction.

- Coil length: Longitudinal length of the coil measured from the lead end to the return end plane.

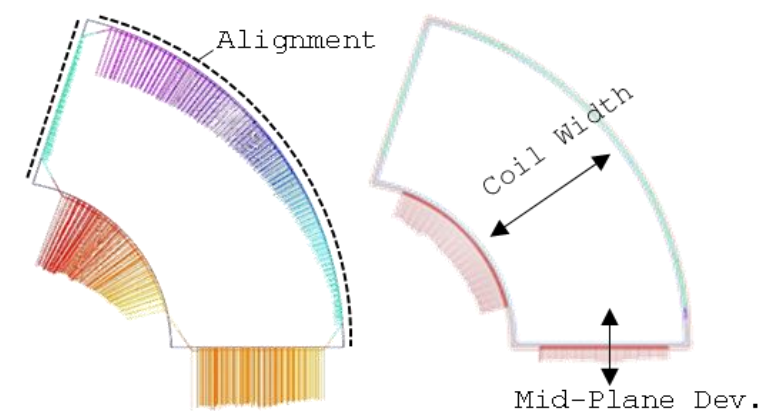

Fig. 3. HL-LHC 11 T Dipole cross section deviation vectors, before (Left Picture) and after (Right Picture) alignment.

\section{PRoduction OvervieW}

The coil geometry is analysed along production with the aim of identifying the main factors driving the manufacturing variability.

Production data is shown in boxplots where whiskers extend to the extreme points and outliers are plotted separately. 


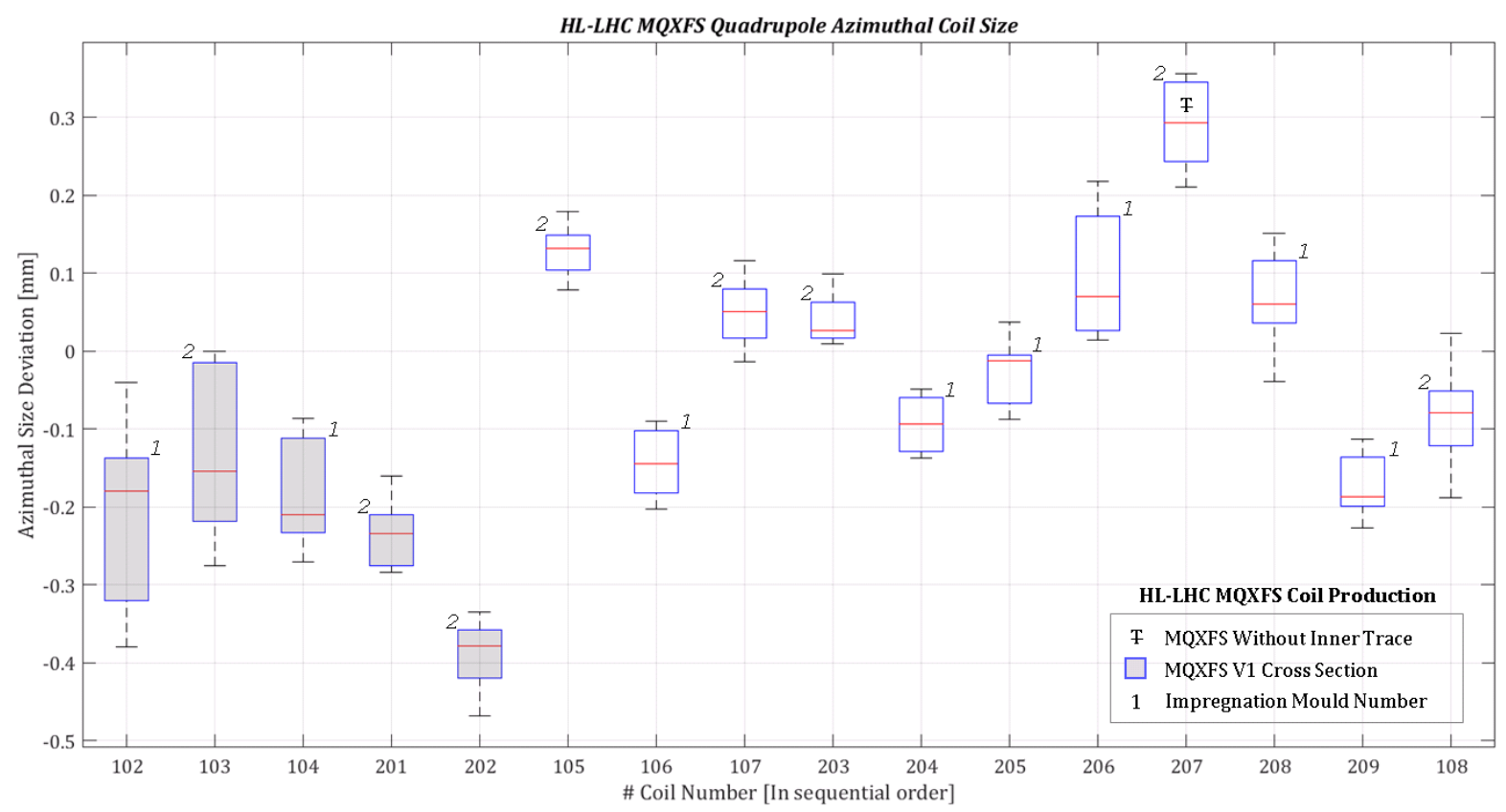

Fig. 4. HL-LHC MQXFS Quadrupole azimuthal coil size during production.

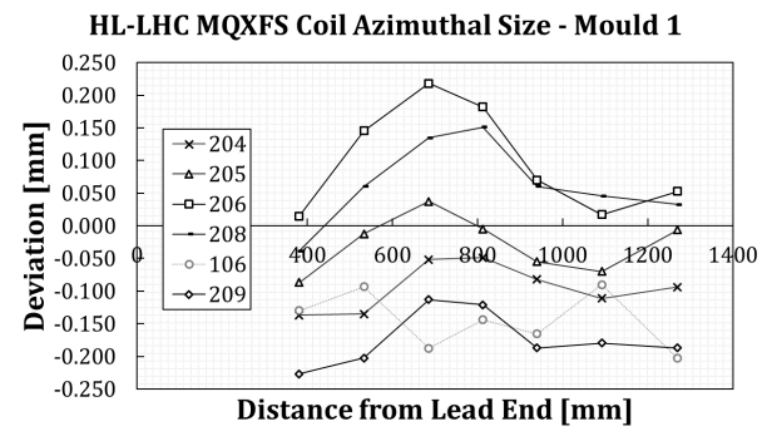

Fig. 5. HL-LHC MQXFS azimuthal coil size for coils impregnated using mould number 1 .

\section{A. The HL-LHC MQXFS Quadrupole}

The coil design of the MQXFS Quadrupole underwent the evolution from a first cross sectional geometry called V1 to the next generation of coils called V2, without modifying the coil external dimensions [11]. For the whole production, the manufacturing process has been always maintained and only two different moulds for impregnation have been used.

Coils are named in sequential order with the following convention:

- Series 100: Coils wound with RRP conductor.

- Series 200: Coils wound with PIT conductor.

\section{1) Coil Size}

In terms of coil size, the first evident sign perceived during production is the increase of the actual size from the first generation cross section to the second one. Fig. 4 shows that V2 cross section coils are generally bigger in azimuthal direction than the V1 ones.

This can be explained by the fact that V2 cross section has been designed to increase the packing of the conductor inside the coil. With respect to the first generation cross section, the radial space used in the tooling to accommodate the cable width expansion has been decreased. On parallel, maintaining
HL-LHC MQXFS Coil Azimuthal Asymmetry Mould 2

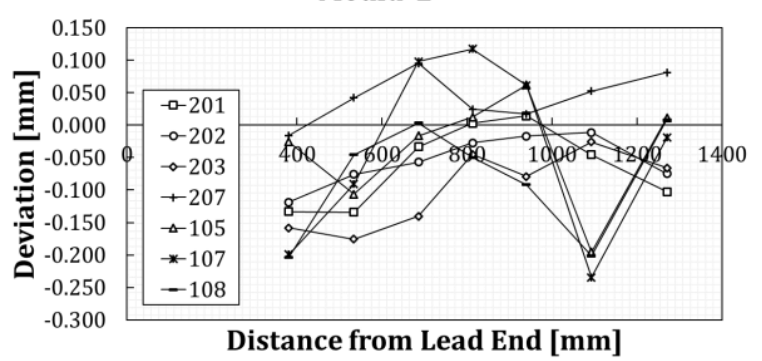

Fig. 6. HL-LHC MQXFS coil asymmetry for coils impregnated using mould number 2 .

the impregnation cavity, the compaction for the inner layer and outer layer turns has been increased by $0.220 \mathrm{~mm}$ and $0.280 \mathrm{~mm}$ respectively. A detailed description can be found in [11].

Analysing the longitudinal variation of azimuthal size as function of the impregnation tooling, there exists a signature of the tooling used. Each mould predominantly prints a characteristic coil size longitudinal variation, although the average size can be different from coil to coil (See Fig. 5).

Regarding the different conductors used, there is no clear impact of the type of conductor on coil azimuthal size.

The non-negligible variations in average coil size, even when the coils were manufactured with the same tooling and cross section version is an evidence that the final geometry and size magnitude strongly depends on each coil production process and variables. It is thought that the manual nature of the full production process, the modular design of the moulds, the necessary tolerances needed for the mould assembly and the final release of internal stresses after mould opening could cause these variations.

An analysis on the impact of the impregnation and reaction tooling tolerances is presented in [12]. The tooling surface tolerance for outer and inner diameters and coil mid-planes is $\mathbf{5 0}$ 
$\mu \mathrm{m}$, but tolerance build-up with the alignment pins allows more variation for coil size and asymmetry $(175 \mu \mathrm{m})$.

\section{2) Coil Asymmetry}

Regarding coil asymmetry, the results are less consistent. They indicate that the same impregnation tooling is able to produce coils with different asymmetry levels and different longitudinal variation, as shown in Fig. 6. It is not possible to link the asymmetry to any identified systematic factor yet. This observation is contradictory to the main findings from [12]. The reason of this difference is that coil asymmetry is governed by the alignment to the coil keyway, and therefore highly affected by the geometry of the pole parts. Important deviations from nominal geometry in some of the used pole parts have been found. The issue is now corrected.

\section{3) Coil Width}

The coil width, measured from the outer surface to the inner one, systematically reproduces the trend shown in the azimuthal size. The coils are generally bigger or smaller both in azimuthal and radial direction at the same time. The tooling signature is also present here.

\section{4) Coil Length}

The coil length is monitored during most of the coil construction steps. The average value for all coils measured at the end of the production process is $1519.074 \mathrm{~mm}$. The dispersion is quantified using the range $(5.278 \mathrm{~mm})$ and standard deviation values $(1.41 \mathrm{~mm})$.

\section{5) Geometry Linked To The Testing Phase}

Important conclusions can be extracted from the analysis of geometrical measurements before and after cold test. The four coils tested in MQXFS3a magnet have been re-measured after the first test. Only information about azimuthal size and coil length is available due to presence of instrumentation in the inner surface of the coils. All coils showed a decrease in azimuthal coil size, maintaining the previous asymmetry level (See Fig. 7 and Fig. 8). The biggest decrease in size is identified for Coil 106, with an average decrement of $60 \mu \mathrm{m}$. Instead, the longitudinal length of the coils has increased, ranging from $300 \mu \mathrm{m}$ for coil 105 to $500 \mu \mathrm{m}$ for coil 107 .

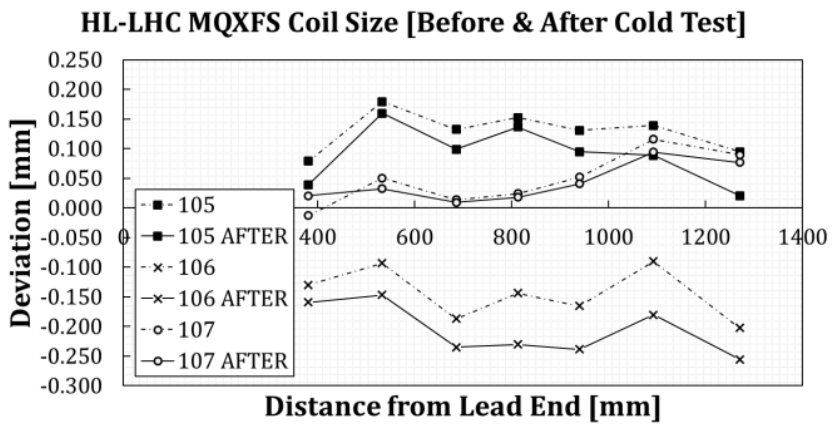

Fig. 7. HL-LHC MQXFS coil size before and after cold test. All coils show a decreased azimuthal size after cold test. The average reduction is $40 \mu \mathrm{m}, 60 \mu \mathrm{m}$ and $10 \mu \mathrm{m}$ for coils 105,106 and 107 respectively.
HL-LHC MQXFS Coil Asym. [Before \& After Cold Test]

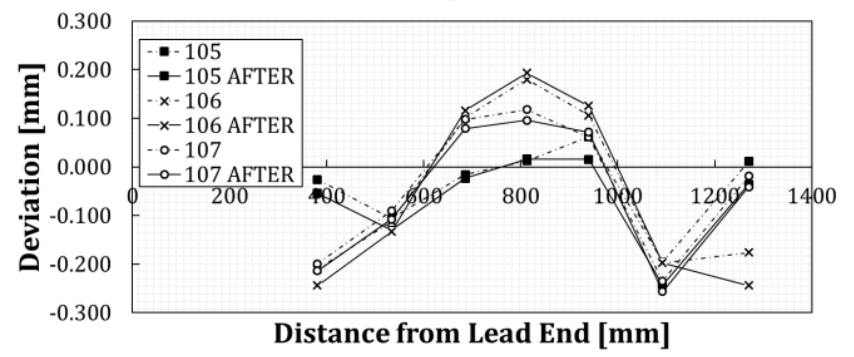

Fig. 8. HL-LHC MQXFS coil asymmetry before and after cold test.

\section{B. The HL-LHC 11 T Dipole}

In the case of the $11 \mathrm{~T}$ Dipole, the production underwent different small adjustments during the development phase: Starting from coil 110, the releasing film located in the impregnation mandrel and outer radius shim has been replaced by a Teflon coating. On parallel, from coil 114, a $100 \mu \mathrm{m}$ thick polyimide trace has been introduced before impregnation. Similar to the HL-LHC MQXFS situation, the nominal external geometry has been always maintained and all modifications were compensated.

\section{1) Coil Size}

Fig. 9 shows the azimuthal coil size for all the measured coils. The spread in azimuthal size and its longitudinal variation is similar in absolute value to the one seen in the HL-LHC MQXFS quadrupole. However, it is important to note that the size of the aperture for the dipole is $1 / 3$ of the quadrupole one. Results then suggest a worse situation in terms of coil geometry for the HL-LHC $11 \mathrm{~T}$ Dipole.

HL-LHC $11 \mathrm{~T}$ model coils have always been bigger in size than the nominal geometry. This phenomena is thought to be linked to the greater compaction of the coils inside the impregnation mould and it is supported by the influence of the increased compaction from V1 to V2 on the cross section of HL-LHC MQXFS.

The tooling signature is less evident than in the previous case. Nevertheless, systematic trends can be found in the longitudinal variation of coil size as function of the production tooling, when the production parameters are preserved (See Fig. 10).

Identical factors driving production variability, as the ones commented for the MQXFS quadrupole, are expected.

\section{2) Coil Asymmetry}

Conversely to the results obtained for the HL-LHC MQXFS quadrupole, the identification of a strong signature coming from the production tooling is clearer for some HL-LHC $11 \mathrm{~T}$ dipole coils, as seen in Fig. 11. Though, this behaviour is not always present and it is not possible to identify a complete systematic trend for all the coils manufactured under the same conditions. 


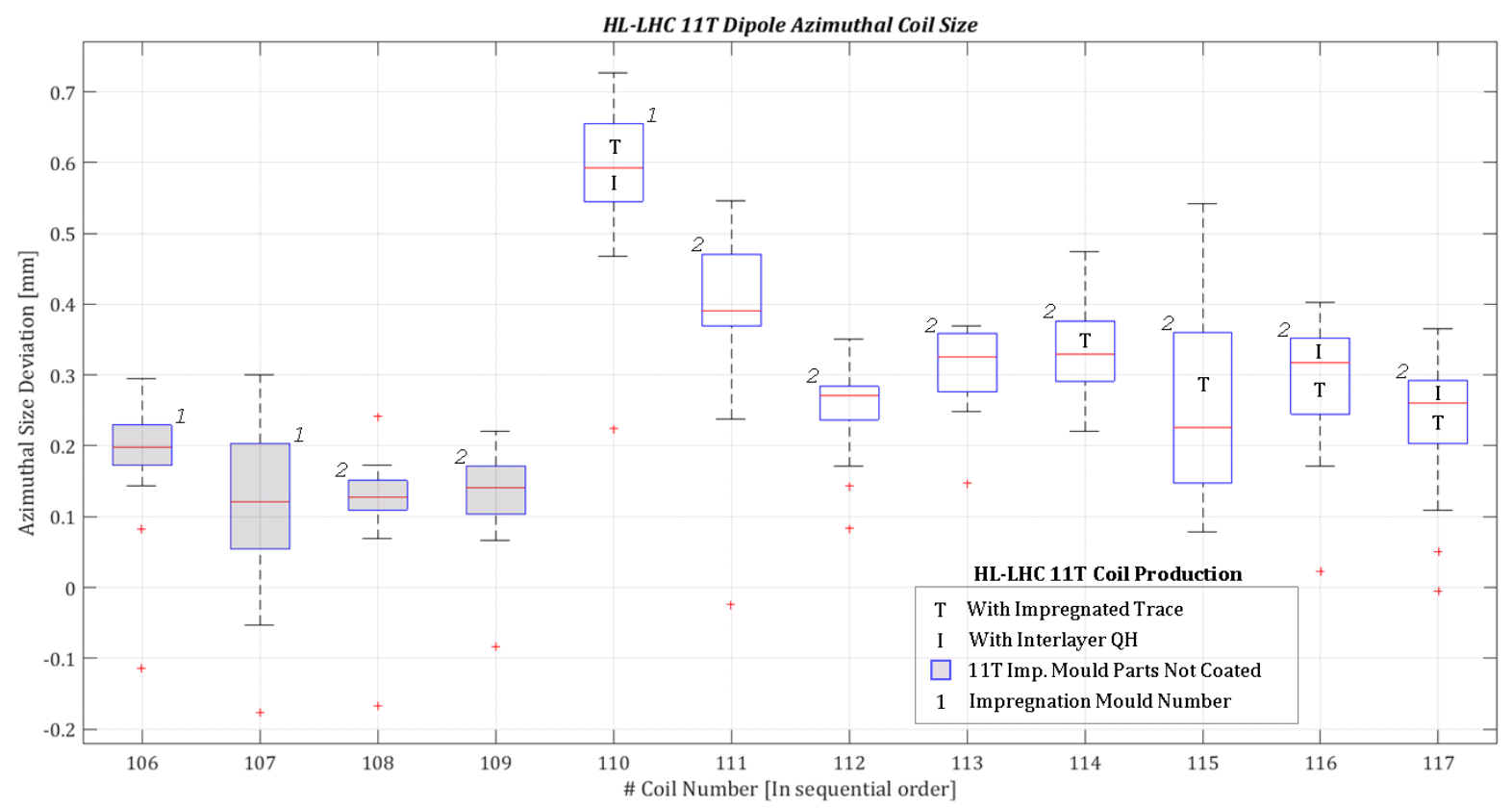

Fig. 9. HL-LHC 11 T Dipole azimuthal coil size during production.

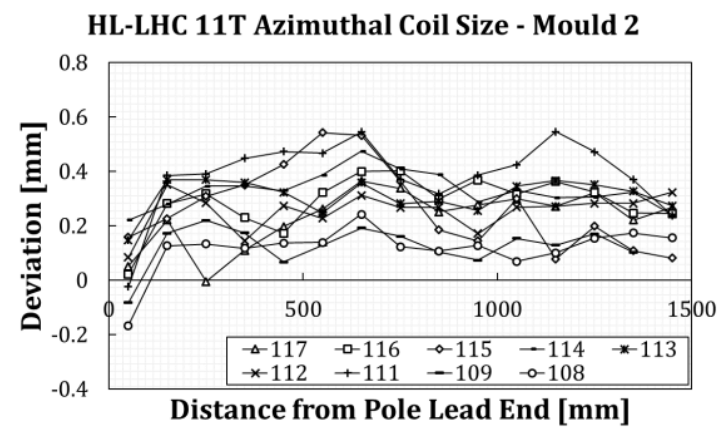

Fig. 10. HL-LHC 11 T Dipole azimuthal coil size for coils impregnated using mould number 2 .

\section{3) Coil Width}

The increment of the coil size is again largely global in radial and azimuthal direction, permanently resulting in a coil cross section that has been globally increased or decreased with respect to the nominal one. The longitudinal variation also reproduces the coil azimuthal size trend.

\section{4) Coil Length}

The average coil length and standard deviation values for the model production are $1974.04 \mathrm{~mm}$ and $1.619 \mathrm{~mm}$ respectively. The longitudinal length range for the HL-LHC $11 \mathrm{~T}$ Dipole model coils equals to $6.3 \mathrm{~mm}$. Results are summarized in Table I.

TABLE I

HL-LHC MODEL COIL LENGTH

\begin{tabular}{ccc}
\hline \hline Magnet & Average $[\mathrm{mm}]$ & Standard Deviation \\
\hline HL-LHC MQXFS & 1519.074 & 1.408 \\
HL LHC 11 T Dipole & 1974.04 & 1.619 \\
\hline
\end{tabular}

HL-LHC 11T Azimuthal Coil Asymmetry Mould 1

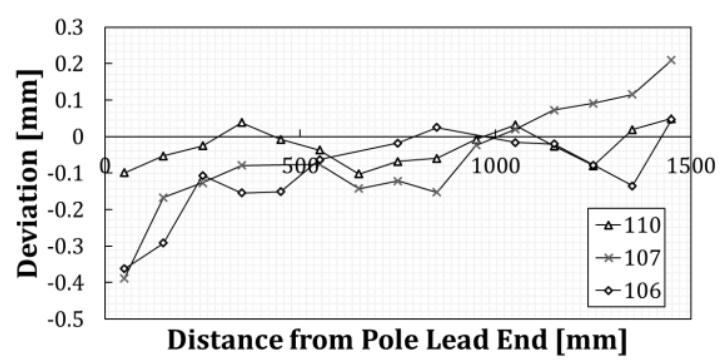

Fig. 11. HL-LHC 11 T Dipole coil asymmetry for coils impregnated using mould number 1 .

\section{CONCLUSION}

The geometrical measurements methodology adopted for the HL-LHC model coils has been explained.

An alignment convention is chosen in order to reproduce the functional behaviour of the coils.

The results show that a remarkable spread in terms of coil geometry is present during the production: For the HL-LHC MQXFS Quadrupole, the average azimuthal size ranges from coil $202(-0.388 \mathrm{~mm})$ to coil $207(0.291 \mathrm{~mm})$. For the HLLHC $11 \mathrm{~T}$ Dipole, the biggest average azimuthal size is found for coil $110(0.577 \mathrm{~mm})$ and the smallest for coil $108(0.116$ $\mathrm{mm})$. The study of this average coil size shows a standard deviation of $0.168 \mathrm{~mm}$ for the HL-LHC MQXFS Quadrupole and $0.132 \mathrm{~mm}$ for the HL-LHC $11 \mathrm{~T}$ Dipole respectively.

The signature of the production tooling can be perceived in the longitudinal variation of coil size. Still, different size magnitudes and asymmetry levels are obtained for the same production configuration. Factors like the stress release after impregnation, production tolerances and production variations are thought to be the reason of the great scattering. 
Globally, the geometric variability is similar in absolute value for both HL-LHC magnets. Changes can be appreciated when modifying the material compaction inside the impregnation mould.

\section{ACKNOWLEDGMENT}

The authors would like to thank all the participants in the construction of model coils for both projects.

\section{REFERENCES}

[1] "Superconducting Accelerator Magnets". K-H Mess, S. Wolff, P. Schmüser (Univ. Hamburg, Germany \& DESY, Germany). World Scientific ISBN: 978-981-02-2790-6.

[2] "LHC Design Report" 0. Bruning, P. Collier, P. Lebrun, S. Myers, R. Ostojic, J. Poole, P. Proudlock. CERN-2004-003, 4 June 2004.

[3] "HL-LHC preliminary design report" Conseil Européen pour la Recherche Nucléaire (CERN), Geneva, Switzerland, CERN-ACC-20140300, Nov. 2014.

[4] L. Rossi and O. Brüning, "High luminosity large hadron collider a description for the European strategy preparatory group," CERN, Geneva, Switzerland, CERN-ATS-2012-236, 2012.

[5] F. Savary et al., "The 11 T dipole for HL-LHC: Status and plan," IEEE Trans. Appl. Supercond.., vol. 26, no. 4, Jun. 2016, Art. no. 4005305.

[6] P. Ferracin et al., "Magnet design of the $150 \mathrm{~mm}$ aperture low- $\beta$ quadrupoles for the high luminosity LHC," IEEE Trans. Appl. Supercond., vol. 24, no. 3, Jun. 2014, Art. no. 4002306.

[7] F. Savary et al., "Progress on the Development of the Nb3Sn 11 T Dipole for the High Luminosity Upgrade of LHC" IEEE Trans. Appl. Supercond., vol. 27, no. 4, Jun. 2017, Art. no. 4003505.

[8] P. Ferracin et al., "Development of MQXF: The Nb3Sn low- $\beta$ quadrupole for the HiLumi LHC," IEEE Trans. Appl. Supercond., vol. 26, no. 4, Jun. 2016, Art. no. 4000207.

[9] "FARO Edge and ScanArm HD: Features, Benefits \& Technical Specifications". Revised 19 July 2016. http://www.faro.com/

[10] "Methods for Performance Evaluation of Articulated Arm Coordinate Measuring Machines (CMM)" ASME B89.4.22 - 2004

[11] S. Izquierdo Bermudez et al., "Second Generation Coil Design of the $\mathrm{Nb} 3 \mathrm{Sn}$ low- $\beta$ Quadrupole for the High Luminosity LHC" IEEE Trans. Appl. Supercond., vol. 26, no. 4, Jun. 2016, Art. no. 4001105.

[12] E.F. Holik et al., "Fabrication and Analysis of 150-mm-Aperture Nb3Sn MQXF Coils" IEEE Trans. Appl. Supercond., vol. 26, no. 4, Jun. 2016, Art. no. 4000907. 